\title{
Micro-Features of Spinel
}

Nathan Renfro, John I. Koivula, Shane F. McClure, Kevin Schumacher, and James E. Shigley

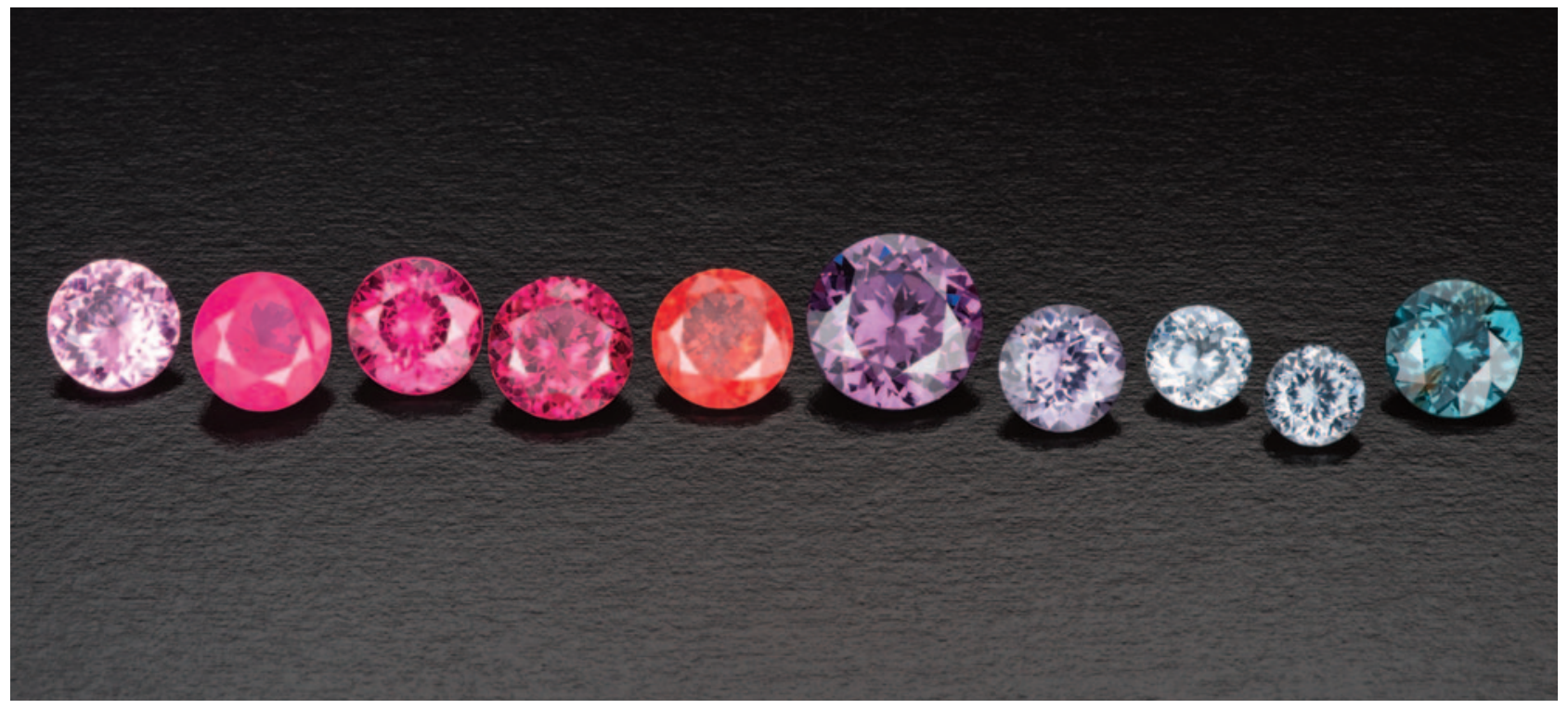

S pinel has long been an underappreciated gem, often placed in the shadows of its traditionally more expensive gemstone cousin, the ruby. Historically, red spinel was visually mistaken for ruby before being acknowledged as its own mineral species, the most notable being the Black Prince's ruby which adorns the Imperial State Crown on display in London. In recent years, spinel has seen a significant rise in popularity and is recognized as one of three birthstones for August. Spinel's appeal may be due in part to its range of colors, from red to pink, purple, blue, gray, and orange (figures 1 and 2).

Spinel is an isometric mineral that often forms as a result of contact metamorphism, finding its home in magnesium-rich dolomite marbles, as is the case with spinel from Myanmar and Pakistan. Other notable spinel sources are Sri Lanka, Tanzania, and Tajikistan.

Gems \& Gemology, Vol. 57, No. 1, pp. 46-49,

http://dx.doi.org/10.5741/GEMS.57.1.46

(C) 2021 Gemological Institute of America
Inclusions found in spinel often reflect the geologic conditions in which they form, with carbonate inclusions (such as calcite) being quite common, as well as apatite crystals. Notably, many spinels contain well-formed octahedral negative crystals that are typically filled with solid minerals, fluids, or a combination of both. Metal sulfides are occasionally present, as well as intergrowths of needle- or plate-like crystals of rutile or sphene that cause asterism.

Treatments for spinel are generally few, with reducing the visibility of fractures by filling them

Figure 1 (above). Spinel is available in a wide range of colors, as seen in this suite of round gems weighing 1.47-6.70 ct faceted by Bill Vance. Photo by Robert Weldon/GIA; courtesy of Vance Gems.

Figure 2 (opposite page). This remarkable suite of spinel gems faceted by Bill Vance shows a wonderful range of color and transparency. The $232.9 \mathrm{ct}$ rough mineral specimen gives clues to the crystal's origin, with remnants of the marble host rock preserved. The faceted red spinels range from 2.47 to $5.87 \mathrm{ct}$. Photo by Robert Weldon/GIA; courtesy of Vance Gems. 


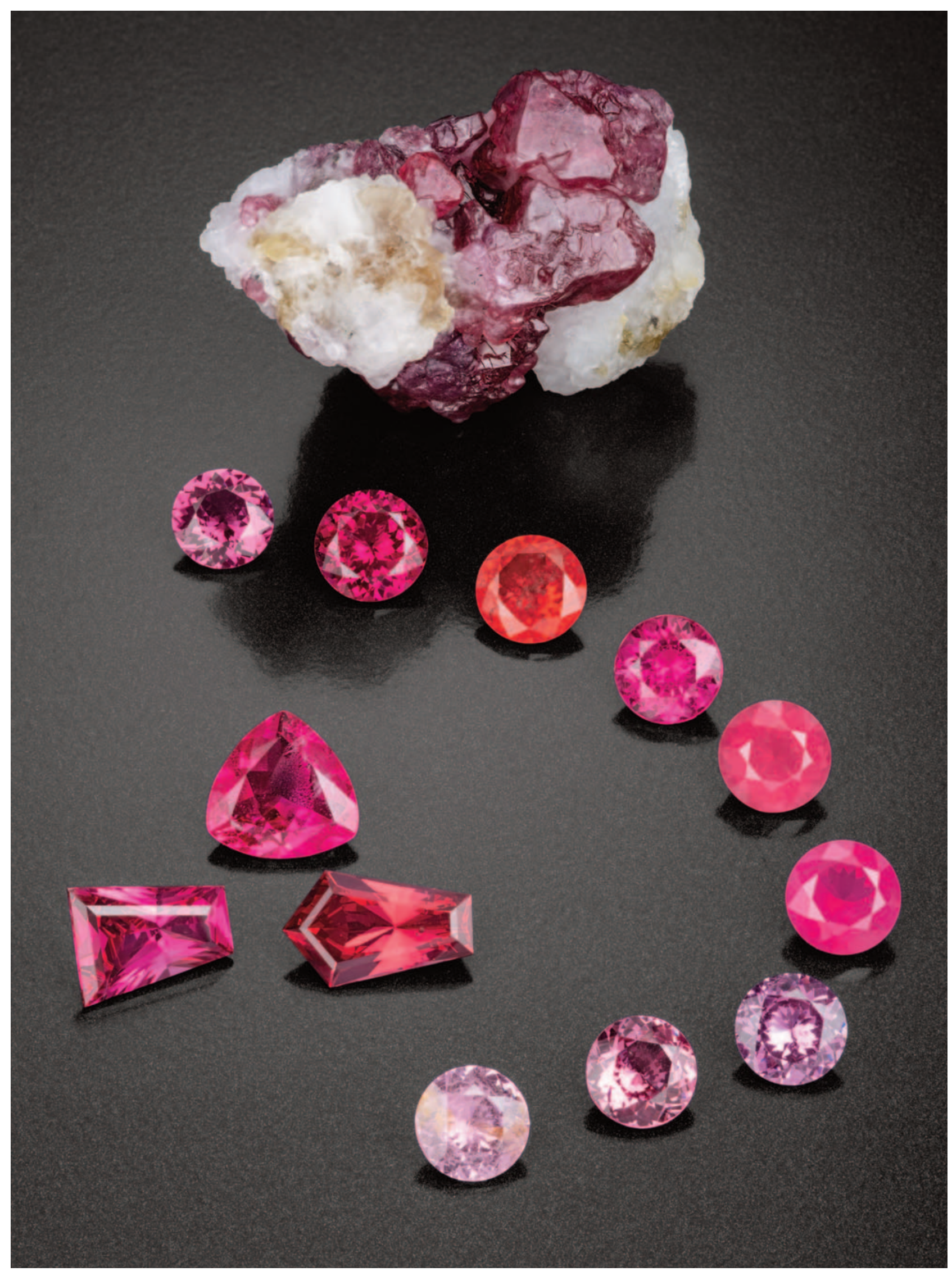


with a refractive index matching material being one most likely encountered as a form of clarity enhancement. Some spinels are also heat treated to improve clarity, but those stones are generally uncommon in the trade; they are readily detectable in a gemological laboratory using photoluminescence or visible spectroscopy, but the treatment often leaves little evidence seen in the microscope. Synthetic spinels are commonly encountered, with flame-fusion material being widely available in a broad range of colors. More technologically sophisticated materials also exist, such as flux-grown

\section{ADDITIONAL READING}

Ahline N., Smith T. (2018) Micro-World: Green crystals in yellow sapphires. Ge G, Vol. 54, No. 1, pp. 70-71.

Altobelli M., Johnson P., Moe K.S. (2013) Lab Notes: Spinel submitted as diamond. $G \uplus G$, Vol. 49, No. 4, pp. 254-255.

Anjomani N. (2016) Lab Notes: Synthetic sapphire and synthetic spinel doublets. $G \uplus G$, Vol. 52, No. 4, pp. 418-419.

Blauwet D. (2011) Gem News International: Spinel from northern Vietnam, including a new mine at Lang Chap. $G \uplus G$, Vol. 47, No. 1, pp. 60-61.

Chauvire B., Rondeau B., Fritsch E., Ressigeac P., Devidal J.L. (2015) Blue spinel from the Luc Yen District of Vietnam. Ge) G, Vol. 51, No. 1, pp. 2-17.

Cooper A., Sun Z.Y. (2014) Lab Notes: Spinel inclusion in spinel. $G \uplus G$, Vol. 50, No. 4, pp. 297-299.

Du Toit G. (2012) Lab Notes: Bicolored spinel. $G \uplus G$, Vol. 48, No. 4, p. 304.

Fryer C. (1991) Lab Notes: Cobalt-colored synthetic spinels with unusual inclusions. Ge G, Vol. 27, No. 2, pp. 113-114.

(1991) Lab Notes: Spinel with unusual green fluorescence. Ge) G, Vol. 27, No. 2, pp. 112-113.

(1996) Lab Notes: Imitation spinel, an unusual assemblage. G»G, Vol. 32, No. 4, p. 281.

Gübelin E.J., Koivula J.I. (1986) Photoatlas of Inclusions in Gemstones. ABC Edition, Zurich.

(2005) Photoatlas of Inclusions in Gemstones, Volume 2. Opinio Verlag, Basel, Switzerland.

Hain M., Sun Z.Y. (2019) Gem News International: Blue gahnite from Nigeria. $G \uplus G$, Vol. 55 , No. 3, pp. 434-436.

Hughes E.B. (2018) Micro-World: Star spinel with four and six rays. G»G, Vol. 54, No. 2, p. 230.

(2020) Micro-World: Actinolite in spinel. GÆ)G, Vol. 56, No. 1, p. 140.

Hughes R.W. (2020) Micro-World: Fluorophlogopite in Burmese spinel. G» G, Vol. 56, No. 2, pp. 293-294.

(2020) Gem News International: Oiled spinel. $G \uplus G$, Vol. 56, No. 3, pp. 443-444.

Huong L.T.T., Hager T., Hofmeister W., Hauzenberger C., Schwarz D., Long P.V., Wehrmeister U., Khoi N.N., Nhung N.T. (2012) Gemstones from Vietnam: An update. $G \uplus G$, Vol. 48, No. 3, pp. $158-176$.

Inns A. (2010) Lab Notes: Synthetic spinel and synthetic ruby doublet. $G \uplus G$, Vol. 46, No. 4, p. 307.

Johnson M.L. (1995) Lab Notes: Spinel, natural with a dendritic inclusion. $G \uplus G$, Vol. 31, No. 1, p. 58 .

_ (1995) Lab Notes: Spinel, with högbomite(?) inclusions. GeG, Vol. 31, No. 4, p. 272.

Johnson M.L., Koivula J.I., Eds. (1996) Gem News: Another tan- spinel. However, those examples are generally available only in red or blue gems or crystals and are relatively scarce.

This latest installment in the $G \uplus G$ chart series showcases a wide range of inclusions that a gemologist might encounter in spinel. Unlike previous charts, however, the authors also chose to highlight a few examples of spinel as an inclusion in other gem materials. Though far from comprehensive, the selected images will hopefully provide the reader with a good representation of the micro-features found in spinel. zanite imitation: Synthetic spinel triplets. $G \uplus G$, Vol. 32, No 4, pp. 289-290.

(1997) Gem News: New information on flux-grown red spinel from Russia. G»G, Vol. 33, No. 2, pp. 151-152.

(1998) Gem News: Spinel from Vietnam. Ge G, Vol. 34, No. 1, pp. 53-54.

(1999) Gem News: Using mineralogical techniques to solve gemological problems, Part 1: Internal "Becke lines" in spinel. $G \uplus G$, Vol. 35, No. 1, p. 58.

Kammerling R.C., Koivula J.I., Johnson M.L, Fritsch E., Eds. (1995) Gem News: Large spinel from Tajikistan. $G \uplus G$, Vol. 31, No. 3, pp. 212-213.

Khoi N.N., Hauzenberger C.A., Sutthirat C., Tuan D.A., Hager T., Nam N.V. (2018) Corundum with spinel corona from the Tan Huong-Truc Lau area in northern Vietnam. Ge G, Vol. 54, No. 4, pp. 404-417.

Koivula J.I., Kammerling R.C., Eds. (1991) Gem News: Tanzanian spinel. Gせ G, Vol. 27, No. 3, p. 183.

Koivula J.I., Kammerling R.C., Fritsch E., Eds. (1992) Gem News: Chinese blue spinels. $G \uplus G$, Vol. 28, No. 1, pp. 61-62.

(1992) Gem News: Blue spinel from Vietnam as sapphire imitator. $G \uplus G$, Vol. 28, No. 2, p. 138.

(1992) Gem News: Update from Sri Lanka. $G \uplus G$, Vol. 28, No. 4, pp. 260-261.

(1993) Gem News: Synthetic spinel from eastern Germany Ge) G, Vol. 29, No. 2, pp. 140-141.

(1993) Gem News: Black spinel from Mexico. GÆ G, Vol 29, No. 3, pp. 212-213.

(1993) Gem News: Spinel from Vietnam. GÆ) G, Vol. 29, No. 3, pp. 213-214.

(1994) "Teal" blue cobalt-colored spinel. GÆ G, Vol. 30, No. 4, pp. 276-277.

Koivula J.I., Kammerling R.C., Johnson M.L., Eds. (1995) Gem News: New gem deposits in Shan State, Myanmar. Ge G, Vol. 31, No. 4, p. 277.

Kondo D., Befi R., Beaton D. (2010) Lab Notes: Heat-treated spinel. Ge)G, Vol. 46, No. 2, pp. 145-146.

Moe K. S. (2011) Gem News International: Trapiche spinel from Mogok, Myanmar. GÆG, Vol. 47, No. 4, pp. 329-330.

Moses T., Reinitz I., McClure S.F. (1998) Lab Notes: Spinel with a darker core. $G \uplus G$, Vol. 34, No. 4, pp. 288-289.

Muhlmeister S., Koivula J.I., Kammerling R.C., Smith C.P., Fritsch E., Shigley J.E. (1993) Flux-grown synthetic red and blue spinels from Russia. $G \uplus G$, Vol. 29, No. 2, pp. 81-98.

Nicastro I., Renfro N., Sun Z.Y., Palke A. (2020) Micro-World: Spinel in sapphire. $G \oplus G$, Vol. 56, No. 3, pp. 433-434.

Overton T.W., Shen A.H. (2011) Gem News International: Cobalt 
blue-colored spinel from Khuoi Ngan, Vietnam. Ge G, Vol. 47, No. 4, pp. 328-329.

Palke A.C. (2019) Gem News International: Gray spinel—A new trend in colored stones. $G \uplus G$, Vol. 55, No. 1, p. 130

Palke A.C., Sun Z.Y. (2018) Symposium Proceedings: What is cobalt spinel? Unraveling the causes of color in blue spinels. $G \uplus G$, Vol. 54, No. 3, p. 262.

Pardieu V. (2014) Hunting for "Jedi" spinels in Mogok. Gせ) G, Vol. 50 , No. 1 , pp. $46-57$

Pardieu V., Long P.V. (2010) Gem News International: Ruby, sapphire and spinel mining in Vietnam: An update. $G \uplus G$, Vol. 46, No. 2, pp. 151-153.

Pardieu V., Sangsawong S., Vertriest W., Raynaud V. (2016) Gem News International: "Star of David" spinel twin crystal with multiphase inclusions from Mogok. $G \uplus G$, Vol. 52, No. 1, pp. $100-101$.

Pay D. (2015) Gem News International: Exceptional red spinels and fine aquamarine. $G \uplus G$, Vol. 51, No. 1, pp. 73-74.

Pham V.L., Pardieu V., Giuliani G. (2013) Update on gemstone mining in Luc Yen, Vietnam. Gせ G, Vol. 49, No. 4, pp. 233245.

Raynaud V., Vertriest W. (2016) Micro-World: Etch marks, negative crystals, and etch tubes in spinel from Madagascar. $G \uplus G$, Vol. 52, No. 3, pp. 313-314.

Renfro N., Koivula J.I. (2015) Micro-World: Violetish blue spinel in yellow sapphire. $G \uplus G$, Vol. 51, No. 4, p. 444.

(2019) Micro-World: Lazurite in spinel. GÆ G, Vol. 55, No. 1 , pp. 112-113

Renfro N., Laurs B.M. (2010) Gem News International: Spinel from Bawma, Myanmar. GeG, Vol. 46, No. 2, p. 154.

Rizzo J. (2019) Lab Notes: Large faceted gahnospinel. GeG, Vol. 55, No. 1, pp. 92-93.

Saeseaw S., Khowpong C. (2016) Lab Notes: Quench-crackled blue synthetic spinel. $G \uplus G$, Vol. 52, No. 1, p. 76

Segura O., Lulzac Y., Fritsch E. (2012) Gems News International: True red synthetic spinel grown by a pulled technique in Russia. $G \uplus G$, Vol 48, No. 3, pp. 232-233.

Sehgal A., Girma D. (2016) Lab Notes: Unusual yellowish green spinel. $G \uplus G$, Vol. 52, No. 2, pp. 194-195.

Stone-Sundberg J. (2019) Gem News International: A variety of colored stones from Mayer \& Watt. $G \uplus G$, Vol. 55, No. 1, p. 139.

Verma P. (2014) Gem News International: Unusual short-wave UV reaction in synthetic blue spinel. $G \uplus G$, Vol. 50, No. 4, pp. 312313.

Vertriest W., Pardieu V. (2016) Update on gemstone mining in northern Mozambique. Ge)G, Vol. 52, No. 4, pp. 404-409.

Vertriest W., Raynaud V. (2017) Micro-World: Complex yellow fluid inclusion in red Burmese spinel. $G \uplus G$, Vol. 53, No. 4, pp. 468-469.

\section{Spinel Inclusions Chart}

To purchase a laminated wall chart featuring superbly detailed photomicrographs of internal features observed in natural, treated, and synthetic spinel, go to store.gia.edu or scan the QR code on the right.
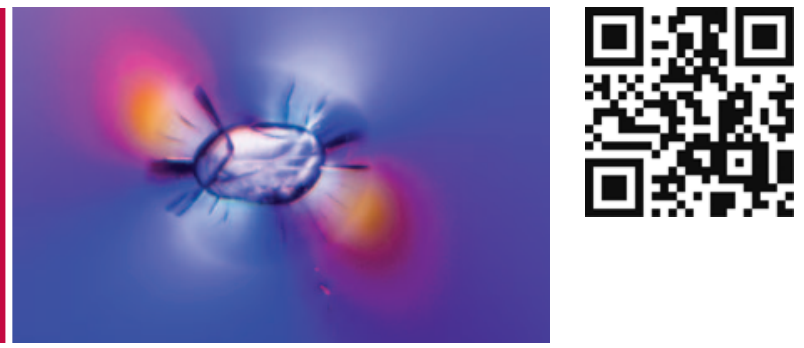